Axel Gehrmann

Der professionelle Lehrer 


\section{Axel Gehrmann}

\section{Der professionelle Lehrer \\ Muster der Begründung - \\ Empirische Rekonstruktion}

Leske + Budrich, Opladen 2003 
Gedruckt auf säurefreiem und alterungsbeständigem Papier.

Die Deutsche Bibliothek - CIP-Einheitsaufnahme

Ein Titeldatensatz für die Publikation ist bei Der Deutschen Bibliothek erhältlich

ISBN 978-3-8100-3803-6

ISBN 978-3-322-90860-5 (eBook)

DOI $10.1007 / 978-3-322-90860-5$

(C) 2003 Leske + Budrich, Opladen

Das Werk einschließlich aller seiner Teile ist urheberrechtlich geschützt. Jede Verwertung auBerhalb der engen Grenzen des Urheberrechtsgesetzes ist ohne Zustimmung des Verlages unzulässig und strafbar. Das gilt insbesondere für Vervielfältigungen, Übersetzungen, Mikroverfilmungen und die Einspeicherung und Verarbeitung in elektronischen Systemen. 


\section{Inhalt}

Vorwort

11

\section{Erster Teil}

\section{Zeit-Läufe:}

Beruf - Professionalisierung - Profession

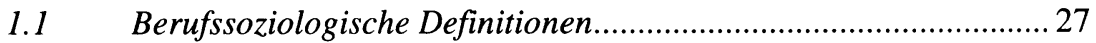

1.2 Erziehungswissenschaftliche Transformationen ............................ 32

$1.3 \quad$ Muster I (historisch - rekonstruktiv) …....................................... 38

\section{Zweiter Teil}

\section{Theorie-Traditionen:}

\section{Von der Autonomie der Pädagogik}

zum lose gekoppelten System

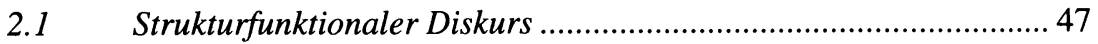

2.1.1 DAS ZUKÜNFTIGE GEHÄUSE DER HÖRIGKEIT ................................ 49

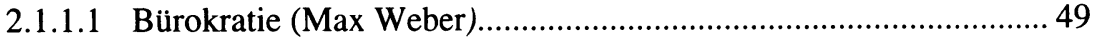

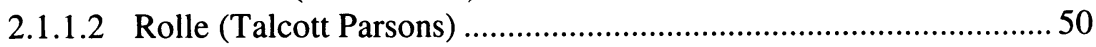

2.1.1.3 System (Niklas Luhmann) ............................................................. 56

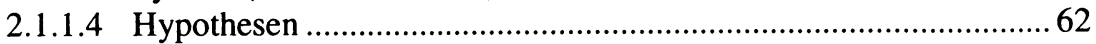

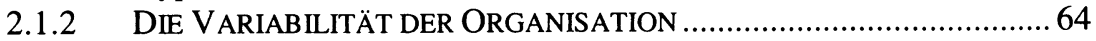

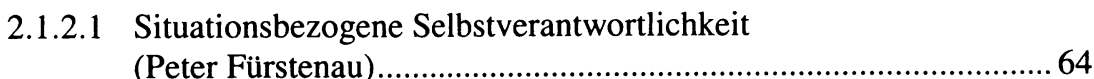

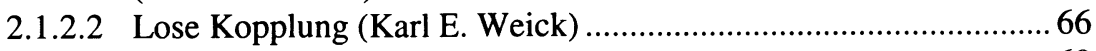

2.1.2.3 Ko-Operation - Mittun (Klaus Türk).............................................69

2.1.2.4 Hypothesen ......................................................................... 73 
2.2 Erziehungswissenschaftlicher Diskurs....................................... 74

2.2.1 DIE KRITIK DER LEHRERROLLE ................................................. 76

2.2.1.1 Pädagogische Selbstrolle (Klaus Mollenhauer) .............................. 76

2.2.1.2 Gesellschaftsharmonistische Einstellungen und mittelständische Ideologien (Arno Combe) ..................................... 80

2.2.1.3 Qualifikationsfunktion - Selektionsfunktion -

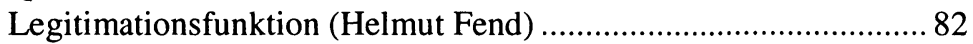

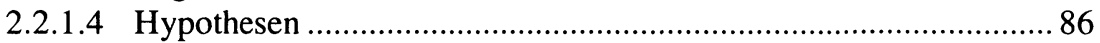

2.2.2 DIE VARIABILITÄT DER PERSON ………....................................... 88

2.2.2.1 Stellvertretende Deutung - Fallverstehen

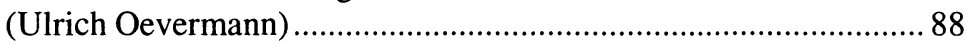

2.2.2.2 Entlastung durch Selbstsorge - Antinomien des Lehrerhandelns (Arno Combe/Werner Helsper) ............................. 94

2.2.2.3 Lehrerpersönlichkeit - Selbstwirksamkeitsüberzeugung (Ulrich Herrmann/Herbert Hertramph) ......................................... 98

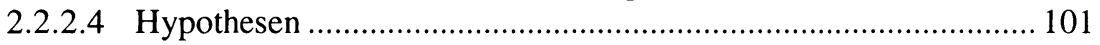

$2.3 \quad$ Muster II (rational - pragmatisch) .............................................. 102

\section{Dritter Teil}

Empirie-Traditionen:

Vom konservativen Wandel zum professionellen Selbst..... 107

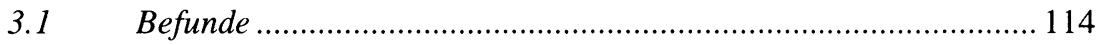

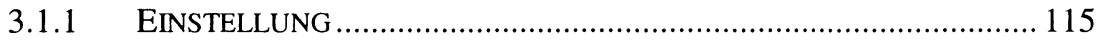

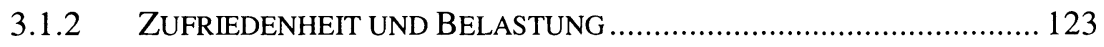

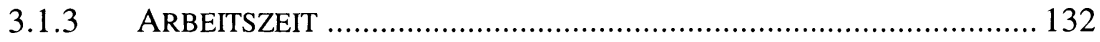

3.1.4 PROFESSIONELLES SELBST .................................................. 138

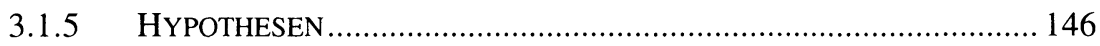

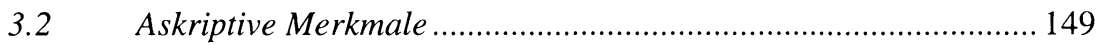

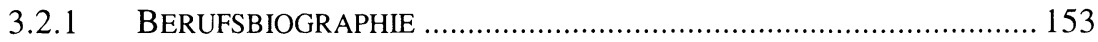

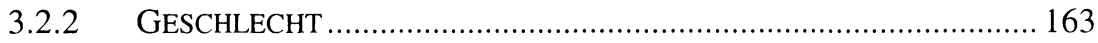

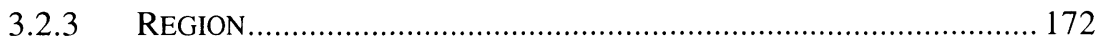

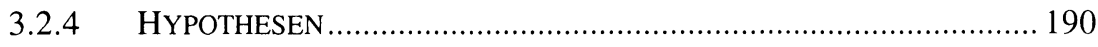

3.3 Muster III (empirisch - analytisch) ....................................... 193 
Vierter Teil

Zeit-Diagnosen:

Vom Verschwinden der Gesellschaft und

der Organisation aus dem Professionsdiskurs

Fünfter Teil

Trend-Beschreibungen:

Von der Singularität des Professionellen

zur Pluralität professioneller Autonomie

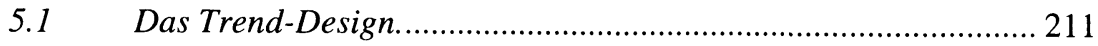

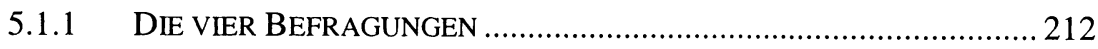

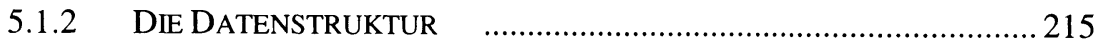

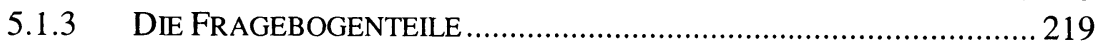

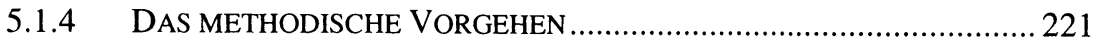

5.1.5 DIE ZENTRALEN HYPOTHESEN......................................................... 225

5.2 Der REGTIME-Datensatz - Die Verbindung aus sieben

Regionen zu vier Zeitpunkten (Berlin 1994, Berlin 1996,

Brandenburg 1998 und Berlin 1999)......................................... 229

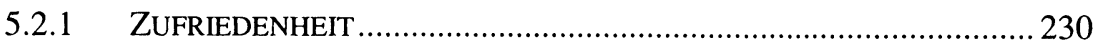

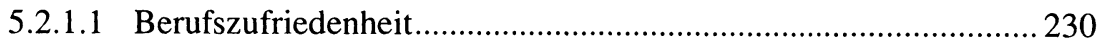

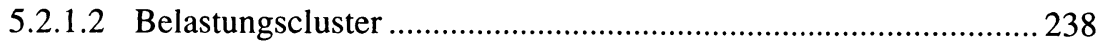

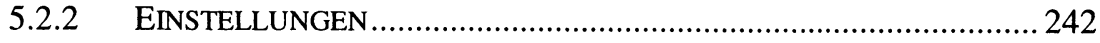

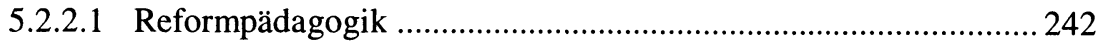

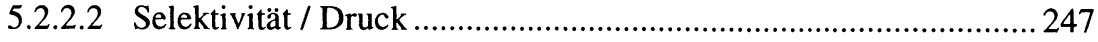

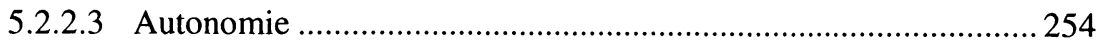

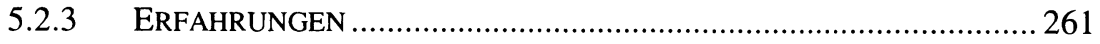

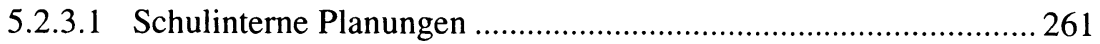

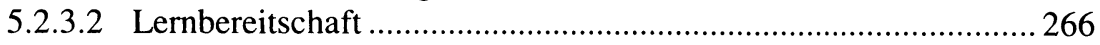

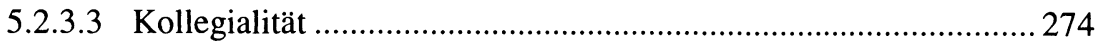

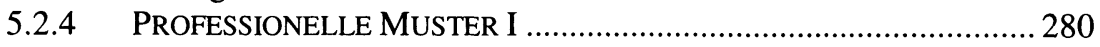

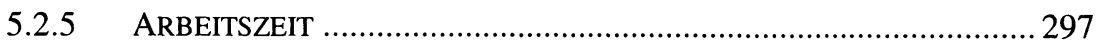

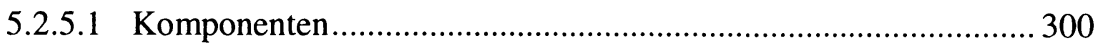

5.2.5.2 Unabhängige Variablen ............................................................ 302

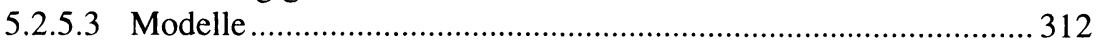

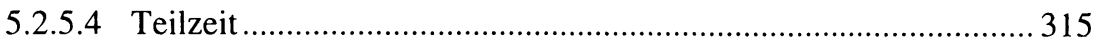


5.3 Der REGTIME3-Datensatz-Die Verbindung aus fün Regionen zu drei Zeitpunkten (Berlin 1996, Brandenburg 1998 und Berlin 1999).

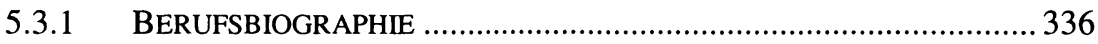

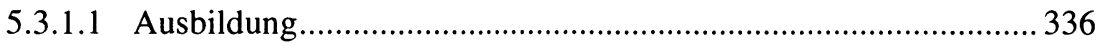

5.3.1.2 Dauer und Unterbrechung der Beschäftigung .............................. 340

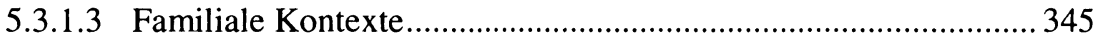

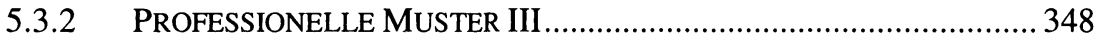

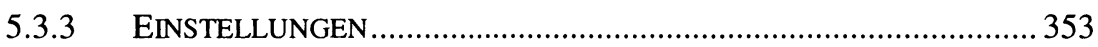

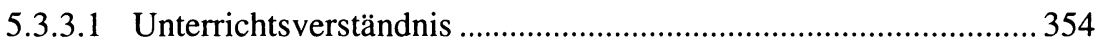

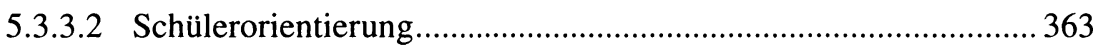

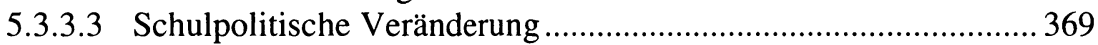

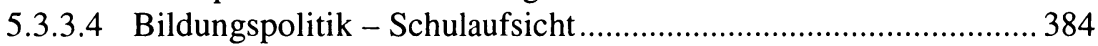

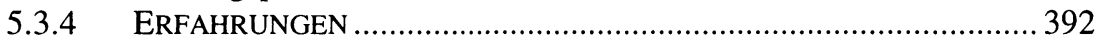

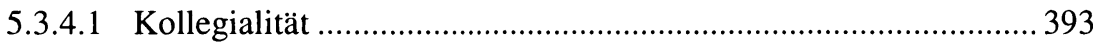

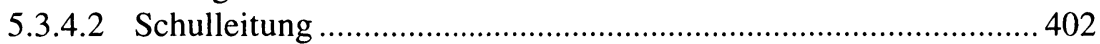

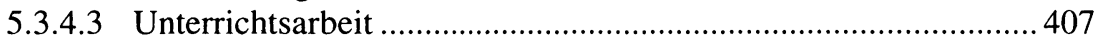

5.3.5 PROFESSIONELLE MUSTER IV ...................................................... 421

Sechster Teil

Zukunfts-Perspektiven:

Von der Abkehr professionstheoretischer

Wandersagen

Literatur 463

Anhang .505 
Den beteiligten Lehrerinnen und Lehrern, die über ihr tägliches Tun Auskunft gaben. 


\section{Vorwort}

Mit dem ausgehenden 20. Jahrhundert endet der deutsche Schulsystemdiskurs der letzten einhundert Jahre. Konstituierte sich im 19. Jahrhundert insbesondere in Preußen ein modernes vertikal und horizontal gegliedertes Schulsystem, und differenzierte sich dieses in der ersten Hälfte des 20. Jahrhunderts weiter aus, so erreichte die damit verbundene Bildungsexpansion nach dem II. Weltkrieg in der Bundesrepublik Deutschland immer neue Höhepunkte.

Der Beitritt der Deutschen Demokratischen Republik unter das Dach des Grundgesetzes im Oktober 1990 zeigt seitdem in den neuen Bundesländern, wie die dort fünfundvierzig Jahre unterdrückte Bildungsexpansion innerhalb weniger Jahre nachgeholt wird. Ein mittlerer Schulabschluss gilt nun überall in Deutschland als Grundbedingung für die Möglichkeit, im Beschäftigungssystem Aufnahme zu finden. Mehr noch, die höchsten zu vergebenden Zertifikate des föderalen Schulsystems sind seit einigen Jahren kein knappes Gut mehr. Immer mehr Schüler erreichen immer hőhere Abschlüsse. Das heutige System aus Haupt-, Real- und Gesamtschulen sowie Gymnasien hat damit genau den Effekt erzeugt, der auch schon am Ende des 19. Jahrhundert sichtbar wurde. Der Versuch, durch immer ausgeklügeltere Schulformen und unterschiedliche Berechtigungen den Zugang zum Gymnasium zu begrenzen, scheitert. Historisch führte dies immer zur Egalisierung auf höherem Niveau. Egalisierung hieße heute, Abitur in Großstädten für die Hälfte und in Flächenländern für $40 \%$ eines Altersjahrganges.

Noch zeichnet sich jene Perspektive erst am Horizont ab, doch deren Ausläufer sind schon deutlich zu spüren. Auf der einen Seite nimmt die Differenzierung des gegliederten Systems insbesondere in der Fläche eher ab, auf der anderen Seite nimmt sie im höchsten Abschlusssegment insbesondere in den Großstädten deutlich zu. So gründeten die neuen Bundesländer (mit Ausnahmen) nur noch sogenannte Mittel-, Sekundar- und Regelschulen, die mittlere Abschlüsse und Gymnasien, die die höchsten Abschlüsse vergeben. Dieses Modell findet auch in den alten Bundesländern schon Anklang. In allen Großstädten mit einem Schulmarkt hingegen sind immer ausdifferenziertere Gymnasialprofile unübersehbar. Beide Prozesse bewirken ein gleiches: 
der Run auf Gymnasien nimmt nicht ab. Alle Schulen, die nicht zum Abitur führen, werden so zu Auffangbecken für diejenigen Schuler, denen der Übergang versagt blieb.

Nachdem in Deutschland über einhundert Jahre ein vertikal und horizontal gegliedertes Schulsystem für den Inbegriff einer modernen Gesellschaft gehalten wurde, verabschiedet sich diese Vorstellung heute und mündet in einen Schulmarkt von Einrichtungen, von denen viele das Abitur vergeben. Da das Gut damit nicht knapp ist, erzeugt der Markt gleichsam aus sich heraus Kriterien, die Knappheit wieder erzeugen beziehungsweise suggerieren. So beginnt die Suche nach den vermeintlich attraktivsten Sek-II-Schulen. Oder umgekehrt: Jede Schule ist gehalten, die vermeintlich attraktivsten Schüler an sich zu binden, um vom Markt nicht abgehängt zu werden.

Das Ergebnis dieser Entwicklung bleibt bis dato offen. $\mathrm{Zu}$ vermuten ist jedoch, dass diese Blickverengung die abgebenden Primarschulen bzw. aufnehmenden Sekundarschulen selbst einem unabweisbaren Druck aussetzen wird. Denn zum einen werden die politisch Verantwortlichen öffentlich nichts unversucht lassen, die Übergänge zu begrenzen, um weitere Expansion einzudämmen, gleichzeitig werden Eltern versuchen, gerade von dieser Expansion nicht abgekoppelt zu werden. Im Fokus dieser Entwicklung stehen dabei letztlich nicht die einzelnen Schulen als Nukleus, sondern diejenigen, die von jeher für die Übergangsentscheidungen (qua Selektion nach Leistung) verantwortlich waren und für diese von der staatlichen Gemeinschaft alimentiert werden, die Lehrerinnen und Lehrer.

Die Erziehungswissenschaft hat in den letzten vierzig Jahren nicht wenig dazu beigetragen, den Schulsystemdiskurs nachzuzeichnen. Die Bundesrepublik wurde in der Nachkriegszeit zunächst aufgeschreckt von der vermeintlich drohenden Bildungskatastrophe, dernach sie vom internationalen Wettbewerb abgekoppelt würde, so sie einer Anstrengung um eine erhöhte Bildungsbeteiligung nicht nachkäme (Carnap/Edding 1962, Picht 1964). Bildungsnotstand ist wirtschaftlicher Notstand, so hieß es. Die Bildungsexpansion war hingegen schon längst im Gange (Hüfner/Naumann 1967).

Fast gleichzeitig eröffnet die erste Nachkriegsgeneration in Deutschland den Diskurs über Chancenungleichheit im Bildungssystem, der universitäre Unterstützung erfährt. Bildung ist Bürgerrecht wird zu einer Metapher der außerparlamentarischen Opposition. Ralf Dahrendorf verwies damit auf die Diskrepanz zwischen Verfassungsnorm und Verfassungswirklichkeit in einer Gesellschaft, die sich schon fast zwanzig Jahre vorher ein Grundgesetz gegeben hatte, indem er betonte, rechtliche „Chancengleichheit bleibt ja eine Fiktion, wenn Menschen auf Grund ihrer sozialen Verflechtungen und Verpflichtungen nicht in der Lage sind, von ihren Rechten Gebrauch zu machen" (Dahrendorf 1965, 24). Er rekurrierte damit auf die Benachteiligung nach Re- 
ligion, sozialem Status, Geschlecht und Region, für die das katholische Arbeitermädchen vom Lande zum Synonym wurde.

In einem bis heute nicht mehr nachvollziehbaren Konsens über alle parteipolitischen Grenzen und allen standespolitischen Lobbyismus erlebt die Bundesrepublik in Folge eine Bildungsreformära, die erst in den 1970er Jahren zum Erliegen kommt. Diese Ära begründet aber nicht nur den Ausbau von integrierten und höheren Schulen, sondern auch von Hochschulen und Disziplinen, die bis dato eher als randständig im universitären Konzert zu bezeichnen sind. Die Erziehungswissenschaft nimmt sich im Zuge ihrer realistischen Wendung (Roth 1962) und am Ausgang ihrer geisteswissenschaftlichen Epoche (Dahmer/Klafki 1968) des Schulsystemdiskurses an, beschreibt die Ungleichheitslagen (Peisert 1967), ruft zur Kritik daran auf (Mollenhauer 1968), evaluiert die neuen (integrierten) Schularten im Vergleich zum gegliederten System (Fend 1982; Blankertz 1986) und ist fast schon dabei, ihre wissenschaftliche Reputation durch Handlungsforschung wieder zu verspielen (Klafki et al. 1982).

Ende der 1970er Jahre, der bildungspolitische Konsens war längst aufgekündigt, steht die expandierte Erziehungswissenschaft ernüchtert vor den Ergebnissen ihrer Diskursbeteiligung. Weder hatten sich die Ungleichheitslagen egalisiert (obwohl hier noch die deutlichsten Fortschritte zu verzeichnen waren, insbesondere durch die Bildungsbeteiligung der Mädchen), noch hatte die Kritik das System aus den Angeln gehoben, geschweige die neuen Institutionen bessere Alternativen geboren. So hieß es bei Fend nüchtern bilanzierend über die Gesamtschulbeobachtung und die Chancenungleichheit, „daß es den Gesamtschulen in bemerkenswertem Maße gelungen ist, Chancenungleichheiten $\mathrm{zu}$ reduzieren und insbesondere Chancengleichheit, gemessen am Kriterium der bedingten Chancengleichheit, also der Chancengleichheit bei Gleichstellung von Intelligenz und Schulleistungen, annähernd zu erreichen" (Fend 1982, 154). Das größte Handlungsforschungsprojekt, das die Bundesrepublik in Bezug auf die Schule je gesehen hatte, wurde nur durch einen Kraftakt beendet. Die zu erforschende Praxis und die darin agierenden Akteure erwiesen sich widerständiger als alle kritisch-konstruktive Theorie und die sie vertretenen Wissenschaftler (Klafki et al. 1982).

Fast scheint es, als habe die ernüchternde Beteiligung am Systemdiskurs am Ende der achten Dekade des 20. Jahrhunderts die Erziehungswissenschaft geradezu in einen neuerlichen Systemdiskurs hineingeführt, als wäre das Stück geblieben, nur die Besetzung habe sich geändert. Es beginnt die Hochzeit der Systemtheorie (Luhmann 1979). Konstatiert wird die Unmoglichkeit von Erziehung, ihre Selbstreferenzialităt und das Technologiedefizit der Pădagogik. Jetzt wird nicht mehr versucht, das Schulsystem beobachtend zu verändern, sondern nur noch beobachtet und die überzeitliche Eigenstruktur 
beschrieben. Dies funktioniert ästhetisch (Mollenhauer 1983, 1986) oder historisch. Dabei zerfällt die historische Bildungsforschung sodann in zwei Teile: die Disziplin- und Professionsforschung (Tenorth 1977) und die historische Schulsystemforschung (Müller 1977). Beiden inhärent ist Empirie, nicht hermeneutische Interpretation von Texten und Bildern.

Die Antwort auf den Systemdiskurs ist quasi eine historisch argumentierende neuerliche realistische Wendung, die auch in der evaluierenden empirischen Bildungsforschung ihr funktionales Äquivalent findet. So trennen sich deren Protagonisten sukzessive von Vergleichsstudien zwischen dem gegliederten und integrierten Schulsystem mit der Begründung, dass die „Systemfrage, die Einschätzung der Bedeutung von Gesamtschulen im Vergleich zum herkömmlichen Bildungswesen" als abschließend behandelt angesehen werden könnte (Fend 1982, 487), doch die Forschung gut daran täte, in Zukunft „nach der Qualität von einzelnen Schulen“ zu fragen (ebd.). Der Systemdiskurs mündet in den um die gute Einzelschule, die Qualität von Schule, auch unterstützt durch internationale Forschungsergebnisse (Rutter et al. 1980). Schließlich hatte sich immer wieder gezeigt, dass die Varianz von Schulleistungen und das Schulklima zwischen einzelnen Schulen des gleichen Systems größer war als die zwischen den Systemen (gegliedert bzw. integriert). Die Sichtweisen partikularisierten sich also. Aus dem Fokus Schulsystem wird der Fokus Einzelschule, der mittlerweile zwanzig Jahre einen Diskursstrang bildet und Regalmeter an Veröffentlichungen nach sich zog (z.B. Tillmann 1989; Aurin 1990, 1993; Steffens/Bargel 1993; Terhart et al. 1994; Gehrmann 1996a; Fend 1996, 1998).

Gleichzeitig nimmt die empirische Bildungsforschung einen Strang wieder verstärkt auf, der im Systemdiskurs untergegangen zu sein schien und nach der Güte von Schulleistungen in einzelnen Schulklassen fragte (Ingenkamp 1969). So hatte es sich nämlich schon früh gezeigt, dass die Variabilität von Schulleistungen zwischen Schulklassen einer Schule größer ist, als die aggregierte Leistung zwischen Schulen. Der Systemdiskurs wird infolge auch hier partikularisiert (Baumert et al. 1986; Helmke 1988; Weinert et al. 1990; Weinert/Helmke 1996) und mündet in Arbeiten über die Variabilität von unterrichtlichen Interaktionen (Weinert/Helmke 1997). Gleichzeitig führt diese Perspektive am Ende der 1990er Jahre wieder in den Systemdiskurs hinein, denn er fließt letztlich ein in nationale (Lehmann/Peek 1997; Lehmann et al. 1998; Baumert et al. 1999) und internationale Schulleistungsvergleiche (Baumert et al. 1997, 1998, 2000), deren erste Ergebnisse schon die Öffentlichkeit aufrührten, und deren Fortführung neue Unruhe bringen wird.

Obwohl in den 1980er Jahren also die historische und empirische Bildungsforschung in eine realistischere Betrachtung der Schulverhältnisse in Vergangenheit und Gegenwart vordringt, bleibt jedoch ein heute sichtbares 
Desiderat übrig. Eine Beobachtung derjenigen, die in der Institution Schule das Erziehungsgeschäft qua Amt betreiben, entwickelt sich nicht. Weder konkretisiert die historische Bildungsforschung Kriterien und Raster für die Beobachtung von individuellen Entwicklungsverläufen im historischen Prozess, noch die empirische Bildungsforschung eine systematische Beobachtung von hauptamtlichen Pädagogen. Der ehemalige Schulsystemdiskurs der empirischen Bildungsforschung aus Schulartenvarianz, Ungleichheitslagen, Kritik und Vergleich wird auf die Einzelschule, die Klasse, den Unterricht, nicht aber auf die Lehrerinnen und Lehrer heruntergebrochen, obwohl sie es sind, die das Erziehungsgeschäft betreiben.

Zwar bleiben Arbeiten nicht vollständig aus, insbesondere was den sogenannten Praxisschock und den konservativen Wandel junger Lehrer angeht (Cloetta 1975; Arbeitsgruppe Aumeister 1976; Dann et al. 1978; MüllerFohrbrodt et al. 1978; Cloetta/Hedinger 1981), doch eine empirische Lehrerforschung etwa entwickelt sich nicht, bleibt „Nicht-Ereignis“ (Terhart 1995a, 234). Eher entstehen vereinzelte Arbeiten, die sich oft nicht aufeinander beziehen bzw. unterschiedlichste Ergebnisse bei ähnlichen Fragestellungen hervorbringen. Selbst deren Wertvollste werden nach und nach von der Öffentlichkeit vergessen (Niemann 1970; Boos-Nünning 1979; Merz 1979).

So wundert es letztlich nicht, dass, als die in der Berufssoziologie der 1970er Jahre begonnene Professionalisierungsdebatte Anfang der 1990er Jahre neuerlich in die Erziehungswissenschaft eindringt (Dewe et al. 1992), in Bezug auf die Lehrerinnen und Lehrer die Autoren nur mögliche theoretische Reimporte der Berufssoziologie bieten können, aber keine auf die Gegenwart bezogene Empirie geschweige Professionstheorie (Terhart 1992a). Der Schulsystemdiskurs hatte die Erziehungswissenschaft an dieser Stelle gleichsam empirisch erblindet. Die Auseinandersetzung mit der in pädagogischen Institutionen sich vollziehenden Erziehungstatsache war Strukturdebatten, Organisationsanalysen, Funktionen und Vollzügen administrativer Entscheidungen gewichen (Schulze 1995).

Erst eine neuerliche realistische Wendung erbringt Veränderung. In einem paradigmatisch zu nennenden Beitrag über die „Bilanz der empirischen Bildungsforschung" konstatiert einer der Protagonisten der Schulsystemforschung, diese habe zwar präzise Standortbestimmungen vorgelegt, aber damit eben nur Rahmenbedingungen beschrieben, die zwei Wirklichkeitsbereiche, „,die in alter Tradition als genuin pädagogisch zu bezeichnen wären“, weniger ins Blickfeld ließen, „Eltern und Lehrer bzw. die Prozesse des Aufwachsens einer jeweils neuen Generation" (Fend 1990, 705). Zusammenfassend schlussfolgert er daraus für die Zukunft, „daß in den nächsten Jahrzehnten diesen interpersonalen und personalen Prozessen, daß Überlegungen zur optimalen Entwicklung des Menschen und deren Beförderungsmöglichkeiten 
durch gestaltende Erziehung wieder mehr Aufmerksamkeit geschenkt wird“ (ebd., 706).

Im Moment seiner facettereichsten Ausprägung, sei es in den vorfindbaren Schularten, sei es in der erziehungswissenschaftlichen Theoriebildung, verliert der Schulsystemdiskurs endgültig seine Bindungskraft, begründet damit den um die gute Schule und macht den Blick frei auf die dafür notwendigen Lehrerinnen und Lehrer. Die Sichtweise ist ,,pädagogischer" geworden, unmittelbarer auf die optimale Menschwerdung in normativem und erfahrungsbezogenem Horizont ausgerichtet, ohne allerdings die Bezüge zu den gemeinschaftlichen und gesellschaftlichen Rahmenbedingungen (...) zu vergessen“ (ebd.).

Mit dem Beginn der letzten Dekade des 20. Jahrhundert sind in Deutschland sodann Forschungsergebnisse greifbar, die sowohl mit quantitativem als auch qualitativem methodischen Zugriff die berufliche Tätigkeit von Lehrerinnen und Lehrern vermessen. In loser Folge erscheinen nun Arbeiten zur Berufsbiographie (Terhart et al. 1994), zur Zufriedenheit (Ipfling et al. 1995), zur Belastung (Rudow 1994; Bauer/Kanders 1998; Combe/Buchen 1996) und zur geschlechtlichen Differenz (Flaake 1989a; Hänsel 1991, 1992; Brehmer 1992; Koch-Priewe 1997; Horstkemper 2000) bei zunächst westdeutschen, dann auch ostdeutschen Lehrerinnen und Lehrern (Hübner 1994; Riedel et al. 1994; Döbert et al. 1995; van Buer et al. 1995; Gehrmann 1996a; Hoyer 1996; Dirks 1996; Gehrmann/Hübner 1997a, 1997b; Zedler/Weishaupt 1997; van Buer/Squarra 1998; Händle 1998; Böttcher et al. 1999; Gehrmann 1999a). Die askriptiven Merkmale Lebensalter, Berufsalter, Beschäftigungsumfang, das Geschlecht und die Fächerkombinationen, die Schularten und die Regionen werden zu entscheidenden Differenzierungskategorien der in Schulen arbeitenden Akteure. Diese Phase kulminiert in der Gründung eines Jahrbuches für Lehrerforschung (Buchen et al. 1997), fast zwanzig Jahre, nachdem ein Jahrbuch für Schulentwicklung zum ersten Mal erschien (Rolff et al. 1980). Der Schulsystemdiskurs war damit zwar nicht vollständig zusammengebrochen (vgl. schon Rolff 1991), aber ein neuerlicher Professionsdiskurs institutionalisiert, der nicht allein auf die historische Genese hauptamtlicher Pädagogen rekurrierte.

Nüchtern betrachtet lebt dieser Diskurs deutlich von der Betrachtung qualitativer Einzelfälle, denen Repräsentanz in der Realität unterstellt wird (u.a. Flaake 1989a; Buchen 1991; Combe/Buchen 1996; Bauer et al. 1996; Händle 1998; Hertramph/Herrmann 1999; Kunze 1999; Reh 1999a, 1999b). Hypothesen werden damit nicht geprüft. Dies mag noch angehen, wenn darauf nicht abgezielt wird, es wird jedoch ärgerlich, wenn subjektive Theorien für das Ganze genommen werden. Nach der einzelnen Schule wird so der ein- 
zelne Lehrer und sein pädagogisches Tun zum Fokus, nicht ein empirisch greifbares Kollektiv und seine Artikulation, die Lehrerschaft.

Sichtbarster Ausdruck dieser Entwicklung ist der neunhundert Seiten starke Band „Pădagogische Professionalität“ (Combe/Helsper 1996), der den Professionsdiskurs pädagogischer Berufe in den letzten dreißig Jahren nachzuzeichnen versucht, diese Kür aber auch in seiner dritten Auflage noch aufführt, ohne eine einzige Hypothese in Bezug auf die Lehrerschaft empirisch quantitativ zu belegen (1999). Der einzelne Lehrer wird so zum Sprachrohr für ein Kollektiv, pädagogisches Selbst (Bauer et al. 1996) und Selbstwirksamkeitsüberzeugung (Hertramph/Herrmann 1999) das neue Insignium. Eine „distanzierte Analyse der Berufsrealität in allen ihren Dimensionen“ bleibt damit weiter ,noch Desiderat" (Tenorth 1999a, 461).

Die vorliegende Untersuchung knüpft an das Desiderat Berufsrealität von Lehrerinnen und Lehrern an. Sie möchte ein stückweit aufklären über zentrale Dimensionen heutiger beruflicher Tätigkeit in der Institution Schule. Der Fokus hierbei ist das Kollektiv Lehrer, nicht die Entwicklung einiger Individuen daraus. Beschrieben wird eine Arbeit mit der nachwachsenden Generation, die die Gesellschaft einer eigenen Berufsgruppe überträgt und von diesem Prinzip auch nicht abzugehen gedenkt. Seine professionelle Autonomie gewinnt der Lehrer dabei durch diese Übertragung von Verantwortung, die trotzdem immer wieder ein fragiles Gut bleibt, denn sowohl die Gesellschaft und ihre Normen als Ganze als auch die Eltern bergen für den Schulmann, die Schulfrau immer neue (alte) Konfliktlinien, die es auszuhalten, auszutarieren gilt. Niemand wird dabei gezwungen, Lehrer zu werden und sich dieser Belastung zu stellen, doch wenn er dies tut, muss dieser Konflikt ausgehalten werden. Oder, um es mit einem Sprangerschen Diktum aus einem ganz anderen Kontext zu sagen, ,niemand wird gezwungen Lehrer zu werden; aber wer Klavierlehrer werden will, muß Klavier spielen können“" (Spranger 1920, 59).

Der erste Teil der Untersuchung zeichnet den Professionsdiskurs der Erziehungswissenschaft der letzten dreißig Jahre nach. Eingeführt wird in die zentralen Kategorien Beruf, Professionalisierung und Profession und ihre Anwendbarkeit zur systematischen Beobachtung der Tätigkeit von Lehrerinnen und Lehrern.

Im daran anschließenden zweiten Teil wird in den Kapiteln Strukturfunktionaler Diskurs und Erziehungswissenschaftlicher Diskurs der im ersten Teil aufgezeichnete Weg in seine theoretischen Ursprünge verfolgt, mit der Intention, implizite Hypothesen über professionelle Lehrerarbeit zu konfigurieren, die den vorgestellten Diskursen möglicherweise inhärent sind.

Der dritte Teil wendet sich sodann der bis dato nachvollziehbaren empirischen Realität professioneller Lehrerarbeit zu. Hierin kondensiert die 
Kenntnis über Lehrerarbeit in Deutschland in den letzten dreißig Jahren. Dies geschieht sowohl über vorfindbare Paradigmen (Einstellung, Zufriedenheit/ Belastung und Arbeitszeit) als auch askriptive Merkmale (Berufsbiographie, Geschlecht und Region). Explizite Hypothesen lassen sich daraus bilden.

Der vierte Teil der Untersuchung nimmt sich der impliziten und expliziten Hypothesen aus Theorie und Empirie an, bündelt sie zu Mustern der Begründung professioneller Lehrerarbeit und beschreibt so den disziplinären Kanon des Wissens darüber in Deutschland am Ausgang des 20. Jahrhunderts.

Im fünften Teil der Untersuchung werden die vorgestellten Hypothesen an vier standardisierten Lehrerbefragungen aus den Jahren 1994 in Berlin, 1996 in Berlin, 1998 in Brandenburg und 1999 in Berlin geprüft. Dabei handelt es sich jeweils um nach Schulart und Region geschichtete Zufallsstichproben, die sich zu zwei Datensätzen generieren ließen. Im ersten Datensatz (REGTIME, Verbindung aus REGion und TIME) befinden sich alle Items, die über vier Befragungen ab 1994 konstant gehalten werden konnten, im zweiten alle diejenigen, die über die letzten drei Befragungen identisch blieben (REGTIME3).

Der Datensatz REGTIME beinhaltet insgesamt 2958 Fälle mit 111 abhängigen und unabhängigen Variablen. Dies sind 328338 Datenpunkte. Der Datensatz REGTIME3 beinhaltet 2339 Fälle mit 318 abhängigen und unabhängigen Variablen. Dies sind 743802 Datenpunkte. Abzüglich der schon in REGTIME vorhandenen Datenpunkte und eingerechnet der nicht mitgerechneten Dummykodierungen ergibt sich somit ein Datenkorpus von ca. 1 Millionen Datenpunkten aus beiden Datensätzen. D.h., der Datenkorpus ist zehnmal größer als die genauer zu beziffernden Datensätze von Terhart et al. (1994, n=514 mit ca. 160 Variablen: 82240 Datenpunkte) und Ipfling et al. (1995, n=2129 mit 35 Variablen: 74515 Datenpunkte).

Die vier Querschnittuntersuchungen zu unterschiedlichen Zeitpunkten fügen sich zusammen, zumindest für die Berliner Befragungen der Jahre '94, '96 und '99, zu einem Trend-Design, gleich dem ALLBUS, der zweijährig stattfindenden Allgemeinen Bevölkerungsumfrage der Sozialwissenschaften bzw. gleich der vom Institut für Schulentwicklung in Dortmund im zweijährigen Turnus durchgeführten Bevölkerungsumfrage Die Schule im Spiegel der öffentlichen Meinung (mit nicht ganz kongruenten zeitlichen Abständen). Damit lassen sich die in dieser Untersuchung vorgestellten Hypothesen nicht nur an einer Befragung prüfen, sondern an bis zu vier, mit der Option, kollektive Veränderungen aufzuzeigen, denn die größten bisher vorgestellten bundesre- 
publikanischen Lehrerbefragungen basieren jeweils auf einmaligen Querschnitterhebungen unterschiedlicher Stichproben und Fragebogenumfänge ${ }^{1}$.

Die Idee für ein solches Trend-Design stand nicht am Anfang der hier vorgestellten Befragungen, vielmehr entwickelte sich dieses im Laufe der letzten sechs Jahre am Institut für Schulpädagogik und Bildungssoziologie der Freien Universität Berlin. Am Beginn stand eine für die GEW Berlin von Prof. Dr. Peter Hübner durchgeführte Befragung zum Thema Arbeitszeit und Arbeitsbelastung Berliner Lehrer (Hübner 1994, 1995). Hübner beteiligte sich sodann von Ende 1994 bis Mitte 1999 an der Berliner DFG-Forschergruppe Bildung und Schule im Transformationsprozeß von SBZ, DDR und neuen Ländern - Untersuchungen zu Kontinuität und Wandel. In der Projektgruppe 5 ging es dabei um die Transformation der Lehrerrolle in den neuen Bundesländern. Zunächst mit einem qualitativen Design geplant, wurde letztlich auf ein standardisiertes, quantitatives Sample umgestellt.

Für die Befragung 1996 Berufliche Erfahrungen und berufliches Selbstverständnis von Lehrerinnen und Lehrern in Berlin im Rahmen des DFG-

1 Niemann befragte 1965/66 2989 Lehrerinnen und Lehrer im Kontext des Instituts für Internationale Pådagogische Forschung Frankfurt aus Baden-Württemberg, Bayern, Hamburg, Hessen, Niedersachsen und Nordrhein-Westfalen (Niemann 1970). Zur Stichprobe gehörten Volks-, Realschul- und Gymnasiallehrer. 36 Items lagen den Beteiligten zur Beantwortung vor.

Merz befragte 19761253 Bayerische Grund-, Haupt-, Real- und Berufsschullehrer sowie Gymnasiallehrer. 90 Items lagen den Beteiligten zur Beantwortung vor (Merz 1979).

Boos-Nünning veroffentlichte im gleichen Jahr (1979) eine Befragung bei 432 Nordrhein-Westfălischen Grund- und Hauptschullehrern mit 890 (sic!) Items, ohne dass alle ausgewertet worden wăren.

Datenmaterial aus Untersuchungen der 1970er Jahre liegen auch vor bei Kischkel 1979 und Kischkel 1984 mit ca. 145000 Einträgen.

Schümer untersuchte Ende der 1980er Jahre den Medieneinsatz durch Lehrer in der Schule und arbeitete daraus „Unterschiede in der Berufsauffassung von Lehrern und Lehrerinnen" heraus (1992). Ihre Arbeit bezog sich auf $n=4000$, ohne dass die Anzahl der Items beschrieben wurde.

Die vor allem berufsbiographisch orientierte Arbeit von Terhart et al. (1994) aus dem Jahr 1992 basierte auf einer Stichprobe von 514 Niedersåchsischen Lehrerinnen und Lehrern mit ca. 160 Items (Grundschule, Hauptschule, Realschule, Gymnasium).

Die bis dato größte westdeutsche Untersuchung der 1990er Jahre legten schließlich Ipfling/Peetz/Gamsjăger vor (1995). Das Datenmaterial bezog sich auf 2129 Bayerische Grund- und Hauptschullehrer mit 35 Items aus dem Jahr 1990.

Bauer et al. konnten sich schließlich in einer bundesweiten Befragung 1995 zum Thema Burnout auf Antworten von 331 Grundschullehrkrăften und 1123 der Sekundarstufe I beziehen (1998).

Für die ostdeutsche Perspektive liegt neben dem hier vorgestellten Material die Arbeit von Zedler/Weishaupt (1997) aus dem Jahr 1993 vor. 1320 Lehrerinnen und Lehrer wurden an Thüringer Grund- und Regelschulen sowie Gymnasien befragt. Die Arbeit von Hoyer (1996) bezieht sich auf 782 Brandenburger Lehrerinnen und Lehrer aller Schularten aus dem Schuljahr 1993/94. 
Projektes wurde Ende 1995 ein Fragebogen neu konstruiert, der Ergebnisse der Hübner-Befragung aus dem Jahr 1994 bei 655 Berliner Lehrerinnen und Lehrern in Ost und West mit aufnahm ${ }^{2}$. Eine Replikation der Untersuchung fand in der zweiten Genehmigungsphase des DFG-Projektes im Land Brandenburg zwischen Oktober und Dezember 1998 statt. Mit dem Ende des Genehmigungszeitraumes des DFG-Projektes wurde die Idee verwirklicht, die Berlin-Befragung 1996 auch in der Bundeshauptsstadt zu replizieren (Frühjahr 1999) und so letztlich drei Berliner Erhebungszeitpunkte zu generieren $(1994,1996,1999)^{3}$.

Der sechste Teil der Untersuchung konfrontiert schließlich die theoretischen Muster der Begründung professioneller Lehrerarbeit mit dem empirischen Material. Formuliert wird damit ein hypothesengeprüfter Einblick in die Lehrerschaft der Bundesrepublik zum Ende der 1990er Jahre.

Mein Dank gilt an dieser Stelle insbesondere Peter Hübner. Er hat die Idee für das hier vorgelegte Produkt nachdrücklich unterstützt, kümmerte sich um die Genehmigungen und die Finanzen (Dank an den Fachbereich Erziehungswissenschaft und Psychologie und seinen ehemaligen Dekan Heinrich Kemper für die finanzielle Unterstützung des Fragebogendruckes im Jahr 1999). Peter Hübner war in den Feldphasen immer ansprechbar. Die mit einem Trend-Design auftretenden empirischen Probleme konnten mit ihm ausführlich besprochen werden. Unvergessen die gemeinsamen Tage am PC

2 Insgesamt beleuchtete dieser neue Bogen auf 22 Seiten vierzehn Themengebiete mit 384 Items: Fragen zum beruflichen Werdegang und zur gegenwartigen Arbeitssituation (I), Fragen zur Belastung durch die Berufsarbeit sowie zur Berufszufriedenheit (II), Fragen zum Sozialprestige des Berufes im Fremd- und Eigenurteil (III), Fragen zur Autonomie und Heteronomie der Berufsarbeit (IV), Fragen zum Wandel der Berufsarbeit und zu den beruflichen Anforderungen (V), Einstellungen zur Schul- und Bildungspolitik sowie eigene Innovations- und Reformbereitschaft (VI), Fragen zur Kooperation und Kommunikation im Lehrerkollegium (VII), Fragen zur Bedeutung der Schulleitung und dem Verhăltnis zu ihr (VIII), Probleme bei der tăglichen Unterrichtsarbeit sowie der Beziehungen zu den Schulern (IX), normative Interpretationen der Aufgabe von Schule, dem Beruf des Lehrers und den Anforderungen an Schüler (X), Auffassungen über Unterricht und Erziehung in der Schule sowie methodisch-didaktische Orientierungen und deren theoretische wie praktische Begründbarkeit $(\mathrm{XI})$, zum Verhaltnis von Schulverwaltung und Schulaufsicht und deren Aufgaben (XII), zur Rolle von Fort- und Weiterbildung unter dem Gesichtspunkt der Kompetenzerhaltung, der Selbstkontrolle und der Fremdkontrolle (XIII), Fragen zu normativ-ethischen Auslegungen der eigenen Berufsrolle (XIV).

3 Der Autor der hier vorgelegten Untersuchung arbeitete von Dezember 1994 bis November 1995 für ein Jahr als wissenschaftlicher Mitarbeiter im DFG-Projekt und wechselte sodann auf eine Assistentenstelle am Institut für Schulpädagogik und Bildungssoziologie. Er war an der Genese und Umsetzung der Befragung aus dem Jahr 1996 federführend beteiligt, organisierte die Brandenburg-Befragung und Aufarbeitung mit Hilfe einer studentischen Hilfskraft und setzte die dritte Berliner Befragung allein von der Datengewinnung bis zur Datenaufbereitung um. Die hier vorgestellten Trend-Datensătze hat er 1999/2000 erstellt. 
beim „Brüten“ über Ausdrucken und Tabellen der einzelnen Befragungen. Als Institutsleiter stellte er mich wochenlang frei für die Generierung der beiden Datensätze und gab auch in der Schreibphase genügend Zeit.

Peter Hübner starb plötzlich am 12. April 2002 an den Folgen eines kardiologischen Routineeingriffes. Er kann die jetzt der Öffentlichkeit vorgestellte Arbeit leider nicht mehr in den Händen halten.

Herrmann Veith und Jürgen Raschert unterstützten mich durch Gespräche und Rat, wenn im Zuge der Erhebungen einmal mehr Probleme auftraten. Ihr offenes Ohr half über diese Tiefs hinweg. Ich bin ihnen sehr dankbar dafür.

Jürgen Sensch und Thomas Hofsäß hatten von Ferne Anteil am Produktionsverlauf und der Fertigstellung dieser Schrift. Sie waren mir damit eine große Hilfe.

Ohne zwei Aufenthalte in der oberfränkischen Mühle der Familie Hartig im Kulmbacher Land wäre diese Schrift nicht zustande gekommen. Über Wochen war es mir dort in vorlesungsfreier Zeit möglich, die Datensätze zusammenzustellen und nicht darüber zu kollabieren. Mein freundschaftlicher Dank gilt Familie Hartig und insbesondere ihrem Neebschen Anhang.

Meine Familie, insbesondere meine Frau Anke, hat die Last und die Lust dieser Unternehmung wie immer zu spüren bekommen. Vielen, vielen Dank für Zuneigung und Unterstützung. Jetzt liegt vergangene Zukunft hinter uns und wir können uns gemeinsam freuen auf das, was kommt. 